\title{
Scaling behavior of the stationary states arising from dissipation at continuous quantum transitions
}

\author{
Davide Rossini and Ettore Vicari \\ Dipartimento di Fisica dell'Università di Pisa and INFN, Largo Pontecorvo 3, I-56127 Pisa, Italy
}

(Dated: May 26, 2022)

\begin{abstract}
We study the critical behavior of the nonequilibrium dynamics and of the steady states emerging from the competition between coherent and dissipative dynamics close to quantum phase transitions. The latter is induced by the coupling of the system with a Markovian bath, such that the evolution of the system's density matrix can be effectively described by a Lindblad master equation. We devise general scaling behaviors for the out-of-equilibrium evolution and the stationary states emerging in the large-time limit for generic initial conditions, in terms of the parameters of the Hamiltonian providing the coherent driving and those associated with the dissipative interactions with the environment. Our framework is supported by numerical results for the dynamics of a onedimensional lattice fermion gas undergoing a quantum Ising transition, in the presence of dissipative mechanisms which include local pumping and decay of particles.
\end{abstract}

\section{INTRODUCTION}

One of the major challenges of current experimental and theoretical investigations in the field of quantum statistical mechanics and condensed matter physics is the understanding of the out-of-equilibrium dynamics of open many-body systems, arising from coherent Hamiltonian drivings and dissipative mechanisms. The recent technological breakthroughs in the manipulation of atomic and quantum optical systems are paving the way to a careful study of the interplay between the coherent quantum dynamics and the dissipative effects, due to the interaction with the environment [1 4]. The competition between these two mechanisms may lead to stationary states which are not related to a thermalization process, i.e., whose properties are not describable in terms of thermal Gibbs distributions. In particular, novel phenomena may emerge close to a quantum phase transition [5], where the low-energy properties of the system are particularly sensitive to variations of the external conditions.

In this paper we investigate the dynamics of a manybody system in proximity of a continuous quantum transition, in the presence of dissipation arising from the interaction with the environment. We focus on a class of dissipative mechanisms whose dynamics can be reliably described through a Lindblad master equation governing the time evolution of the system's density matrix [6, 7]. This framework is of experimental interest, indeed the conditions for its validity are typically realized in quantum optical and circuit-quantum electrodynamics (c-QED) implementations [1, 2, 8, . We argue that, in the presence of homogenous dissipators, the competition between coherent and dissipative drivings develops dynamic scaling laws involving some relevant parameters of the two mechanisms. This occurs within a low-dissipation regime, where the decay rate of the dissipation is comparable with the gap of the Hamiltonian [9]. General scaling behaviors are put forward, which are expected to be developed along the time evolution described by the Lindblad equation, especially in the large-time limit where stationary states set in. Analogously to the scaling laws of closed systems at quantum transitions, the dynamic scaling behavior in the presence of dissipation is expected to be universal, i.e., largely independent of the microscopic details.

To verify the emerging scaling scenario, we found it convenient to consider, as an example, the paradigmatic Kitaev quantum wire [10]. Namely, we study its dynamic behavior close to the quantum transition, in the presence of dissipation due to local incoherent pumping or decay. Our numerical results support the general dynamic scaling theory, addressing the mutual interplay between coherent dynamics and dissipation at a continuous quantum transition.

Some features arising from the competition of coherent and dissipative dynamics close to quantum transitions were already recently analyzed within a dynamic finite-size scaling framework, see Ref. 9], with specific emphasis on finite systems of linear size $L$, and the dynamic behavior for relatively small times $t \sim L^{z}$ (where $z>0$ is the dynamic critical exponent associated with the quantum transition), thus not including the largetime stationary regime. In this paper we focus on some complementary regimes. Indeed we consider infinite-size systems, for which we derive scaling laws extending to the large-time limit of the evolution described by the Lindblad equation, thus valid for the corresponding stationary states. We shall emphasize that, at this stage, our scaling theory for quantum dissipative systems should be considered as a conjecture arising from phenomenological scaling arguments. Therefore, the scaling behaviors devised for the two mentioned distinct situations are not trivially related, and careful numerical checks are crucial to validate our general framework.

We finally mention that dynamic scaling behaviors have been also put forward, and numerically checked, for open critical systems when the environment is constituted by a single qubit homogeneously coupled to the whole many-body system [11].

The paper is organized as follows. In Sec. III we present the general setup of our dynamic problem, recall- 
ing the scaling laws developed by closed systems at quantum transitions, and the main features of the dissipative mechanisms described by the Lindblad equation. Then, in Sec. III we put forward the scaling laws describing the competition of coherent and dissipative drivings as described by the Lindblad equation, extending the scaling laws of closed systems at quantum transitions to incorporate the effects of the dissipation. In Sec. IV we introduce the one-dimensional Kitaev Fermi model in the presence of dissipation arising from local pumping and decay, and define the observables that we are going to consider, in order to characterize the time evolution of the system, and in particular of the stationary states. Section $\mathrm{V}$ contains and discusses the outcomes of our numerical results, which definitely support the dynamic scaling theory in the presence of dissipation. Finally, in Sec. VI we summarize and draw our conclusions.

\section{CRITICAL SYSTEMS IN THE PRESENCE OF DISSIPATION}

\section{A. Many-body systems at a quantum transition}

We start by summarizing the general scaling features of a many-body system at a continuous quantum transition. Consider a generic $d$-dimensional many-body system with Hamiltonian $\hat{H}$, close to a zero-temperature transition driven by quantum fluctuations [5, 12. A quantum transition is generally characterized by few relevant perturbations, whose tuning gives rise to quantum critical behaviors, characterized by a diverging length scale, and universal power laws.

Let us assume that the system Hamiltonian has one relevant parameter $\mu$, whose tuning toward the point $\mu_{c}$ develops a quantum critical behavior. The critical power laws are generally characterized by the renormalizationgroup dimension $y_{\mu}$ associated with the relevant parameter $\mu$ and the dynamic exponent $z$, so that the diverging length scale of the critical modes behaves as

$$
\xi \sim \lambda \equiv|\bar{\mu}|^{-\nu}, \quad \bar{\mu} \equiv \mu-\mu_{c}, \quad \nu=y_{\mu}^{-1},
$$

and the suppression of the gap (that is, the difference of the two lowest energy levels) as

$$
\Delta \sim \xi^{-z} .
$$

Moreover, the correlation function of generic local operators $\hat{O}(x)$,

$$
G(x ; \bar{\mu}) \equiv\left\langle 0_{\mu}|\hat{O}(x) \hat{O}(0)| 0_{\mu}\right\rangle
$$

where $\left|0_{\mu}\right\rangle$ is the ground state associated with the parameter $\mu$, obeys an asymptotic scaling law of the form [12, 13]

$$
G(x ; \bar{\mu}) \approx b^{-2 y_{\circ}} \mathcal{G}\left(x / b, \bar{\mu} b^{y_{\mu}}\right),
$$

where $b$ denotes an arbitrary positive number and $y_{o}$ is the renormalization-group dimension of $\hat{O}(x)$. The above scaling equation neglects further dependences on other irrelevant Hamiltonian parameters, which are supposed to be suppressed in the large- $b$ limit. Then, if we fix the arbitrary parameter $b$ by requiring

$$
|\bar{\mu}| b^{y_{\mu}}=1
$$

and introduce the variable $\lambda$ as in Eq. (1), we obtain the asymptotic scaling behavior

$$
G(x ; \bar{\mu}) \approx \lambda^{-2 y_{\circ}} \mathcal{G}(x / \lambda)
$$

around $\mu_{c}$. One may also consider equilibrium states at finite temperature $T$, related to a Gibbs distribution of the quantum states. For sufficiently small temperatures, the dependence on $T$ is taken into account [5] by adding a further dependence on $T b^{z}$ in Eq. (4), turning into a dependence on $T \lambda^{z}$ in the scaling equation (6), that is

$$
G(x ; \bar{\mu}, T) \approx \lambda^{-2 y_{\circ}} \mathcal{G}\left(x / \lambda, T \lambda^{z}\right) .
$$

These asymptotic scaling behaviors are expected to be observed in the limit $\lambda \rightarrow \infty$. Their approach is generally characterized by power-law scaling corrections, which may come from different sources [13], such as irrelevant perturbations at the fixed point describing the quantum transition, and analytic corrections in the nonlinear scaling fields entering the scaling laws.

One may also study out-of-equilibrium evolutions close to a quantum transition, for example arising from a sudden quench of the Hamiltonian parameter $\mu$, at $t=0$ from an initial value $\mu_{i}$ to $\mu \neq \mu_{i}$, starting from the ground state at $\mu_{i}$. The corresponding time dependence after the quench can be taken into account within the scaling laws as well, by adding a further dependence on $t b^{-z}$ in Eq. (4). More precisely, the fixed-time correlation functions are expected to behave as [14, 15.

$$
\begin{aligned}
G\left(x, t ; \bar{\mu}_{i}, \bar{\mu}\right) & =\langle\Psi(t)|\hat{O}(x) \hat{O}(0)| \Psi(t)\rangle \\
& \approx \lambda^{-2 y_{o}} \mathcal{G}\left(x / \lambda, t \lambda^{-z}, \bar{\mu}_{i} / \bar{\mu}\right),
\end{aligned}
$$

where $|\Psi(t)\rangle$ is the quantum state after a time $t$ from the quench, and $\bar{\mu}_{i} \equiv \mu_{i}-\mu_{c}$.

The above scaling behaviors have been shown to develop for closed states around the critical point $\mu_{c}$. In the following we study the effects of the presence of dissipation in systems described by critical Hamiltonians.

\section{B. Dissipative interactions}

Suppose that the many-body system is also subject to dissipative interactions with the environment, so that the time dependence of its density matrix $\rho$ is described by the Lindblad master equation [6]

$$
\frac{\partial \rho}{\partial t}=-\frac{i}{\hbar}[\hat{H}, \rho]+u \mathbb{D}[\rho],
$$

where the first term provides the coherent driving, while the second term accounts for the coupling to the environment, characterized by a global coupling constant $u>0$. 
We restrict to homogeneous dissipation mechanisms, preserving translational invariance, as sketched, for example, in Fig. 1. In the case of systems weakly coupled to Markovian baths, the trace-preserving superoperator can be written as a sum of local terms, such as [16, 17.

$$
\begin{aligned}
& \mathbb{D}[\rho]=\sum_{o} \mathbb{D}_{o}[\rho], \\
& \mathbb{D}_{o}[\rho]=\hat{L}_{o} \rho \hat{L}_{o}^{\dagger}-\frac{1}{2}\left(\rho \hat{L}_{o}^{\dagger} \hat{L}_{o}+\hat{L}_{o}^{\dagger} \hat{L}_{o} \rho\right),
\end{aligned}
$$

where $\hat{L}_{o}$ is the Lindblad jump operator associated with the local system-bath coupling scheme, and $o$ denotes an appropriate spatial coordinate. Dissipation mechanisms described by local Lindblad operators $L_{o}$ have been considered in various contexts (see, e.g., Refs. 1, 2, 8, 18, 19]). In several quantum optical devices, in particular in systems with photon leakage or with some qubit relaxation or dephasing, the conditions leading to Eqs. (9)(11) are typically satisfied [1, 2, 8, therefore this formalism constitutes the standard choice for their theoretical investigation. Other interesting implementations in this respect may be provided by hybrid light-matter systems, where atoms are strongly coupled to cavities, thus mediating photon-photon interactions, see e.g. Ref. [19].

In the next section, we are going to address the formation of large-time stationary states arising from the competition of the coherent and dissipative drivings, as described by the Lindblad equation (9). As we shall see below, while the coupling with a bath generally drives the system to a noncritical behavior, even when the Hamiltonian parameters are critical, it is however possible to identify a peculiar low-dissipation regime where the steady state may develop the above mentioned competition and display a critical behavior. To this purpose, we will put forward general scaling behaviors for the stationary states in the low-dissipation regime when the many-body Hamiltonian is within the quantum critical regime, somehow extending the scaling laws reported in Sec. IIA.

\section{SCALING BEHAVIOR OF THE STATIONARY STATES}

Let us assume that, without loss of generality, the quantum many-body system is initialized, at $t=0$, into

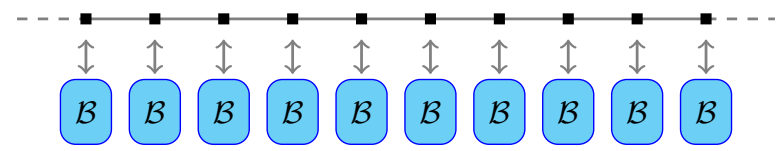

FIG. 1: Sketch of a quantum system on a one-dimensional lattice (black squares), in which different sites may undergo a coherent and uniform nearest-neighbor coupling. Each site is supposed to be homogeneously and weakly coupled to an external and independent bath $\mathcal{B}$ (blue boxes), whose effect is to introduce local incoherent mechanisms. the ground state of the Hamiltonian $\hat{H}\left(\mu_{i}\right)$ for a given parameter $\mu_{i}$. The time evolution for $t>0$ is dictated by the Lindblad equation (9) with the Hamiltonian $\hat{H}(\mu)$, where $\mu$ may differ from $\mu_{i}$, thus realizing a sudden quench, and the system-bath coupling strength is fixed by the coupling $u$. The dissipator $\mathbb{D}[\rho]$ may drive the system to a steady state, which is generally noncritical, even when the Hamiltonian parameters are critical. In the case of dissipation leading to a unique stationary state, the choice of the initial state (that is, the initial state of the time evolution, which is eventually fixed by the parameter $\mu_{i}$ ) is not relevant for the long-time properties of the system; nonetheless it may determine the initial and intermediate time dependence.

We now argue that it is possible to identify a lowdissipation regime, where the dissipation is sufficiently small to compete with the coherent evolution driven by the (critical) Hamiltonian. This leads to a late-time stationary state which can present a critical behavior, depending also on the strength of the system-bath coupling. The effects of a small dissipation are taken into account by adding a further dependence on a scaling variable associated with $u$ in the out-of-equilibrium scaling laws, i.e. $u b^{\zeta}$, where $\zeta$ is a suitable exponent which ensures the substantial balance (thus competition) with the critical coherent driving. Since dissipation is predicted to give rise to a relevant perturbation at the quantum transition, we expect $\zeta>0$. Thus, the peculiar lowdissipation regime outlined above should be characterized by $u \sim \lambda^{-\zeta}$.

As already argued in Ref. [9], the exponent $\zeta$ should generally coincide with the dynamic exponent $z$. This is expected by noting that the parameter $u$ in Eq. (9) plays the role of a decay rate, i.e., of an inverse relaxation time for the associated dissipative process $[6$, and any relevant time scale $t_{s}$ at a quantum transition behaves as $t_{s} \sim \Delta^{-1}$ [14]. In other words, to observe a nontrivial competition between critical coherent dynamics and dissipation, one should consider a sufficiently small coupling $u \sim \lambda^{-z}$, so that its size is comparable with the energy difference $\Delta \sim \lambda^{-z}$ of the lowest energy levels of the Hamiltonian. An analogous conjecture was put forward to describe the approach to thermalization of some specific open systems close to a quantum transition [20]. Here we extend it to more general situations, even when the final stationary state is not thermal.

On the basis of such scaling arguments, generic fixedtime correlation functions in the large-volume limit are thus expected to behave as

$$
\begin{aligned}
G\left(x, t ; \bar{\mu}_{i}, \bar{\mu}, u\right) & =\operatorname{Tr}[\rho(t) \hat{O}(x) \hat{O}(0)] \\
& \approx \lambda^{-2 y_{o}} \mathcal{G}\left(x / \lambda, t \lambda^{-z}, \bar{\mu}_{i} / \bar{\mu}, u \lambda^{z}\right),
\end{aligned}
$$

where $\rho(t)$ is the time-dependent density matrix of the system. We conjecture that this scaling ansatz describes the low-dissipation regime of quenching protocols for many-body systems at quantum transitions. The main features of such dynamic critical regime are expected to 
depend only on the universality class of the transition and the general properties of the dissipative mechanism.

In order to derive the scaling laws for the asymptotic stationary states, we need to consider the large-time limit of the scaling equation (12). Hereafter we shall assume that the asymptotic stationary state is unique, i.e., independent of the initial conditions of the protocol that we consider, as is the case for several classes of dissipators 21 24]. Such stationary state should appear when $t \gg \lambda^{z}$. Therefore, we conjecture the scaling law

$$
\begin{aligned}
G_{s}(x ; \bar{\mu}, u) & \equiv G\left(x, t \rightarrow \infty ; \bar{\mu}_{i}, \bar{\mu}, u\right) \\
& \approx \lambda^{-2 y_{o}} \Gamma\left(x / \lambda, u \lambda^{z}\right),
\end{aligned}
$$

where in the definition of $G_{s}$ we assumed that the dependence on the initial parameter $\bar{\mu}_{i}$ drops, under an assumption of unicity of the stationary state.

It is also possible to define a correlation length $\xi_{s}$, from the exponential large-distance decay of the correlation function $G_{s}$. Indeed, since we expect that the largedistance behavior $G_{s} \sim e^{-x / \xi_{s}}$, we may define

$$
\xi_{s}^{-1}(\bar{\mu}, u)=-\lim _{x \rightarrow \infty} \frac{\ln G_{s}(x ; \bar{\mu}, u)}{x} .
$$

Using the scaling laws derived for $G_{s}$, we obtain

$$
\xi_{s} \approx \lambda \mathcal{L}_{1}\left(u \lambda^{z}\right),
$$

or alternatively

$$
\xi_{s} \approx u^{-1 / z} \mathcal{L}_{2}\left(u \lambda^{z}\right) .
$$

Note that this scaling equation is formally analogous to that obtained at the thermodynamic equilibrium, after replacing $u$ with the temperature $T$, see Sec. II A and in particular Eq. (7). However, we stress that our arguments extend to cases where the asymptotic stationary state does not coincide with a Gibbs equilibrium state. This will be indeed the case for the Kitaev model subject to incoherent particle decay or pumping [see Eq. 20 and the discussion below].

We finally stress that the strictly local (on-site) nature of the Lindblad operators is not essential for the dynamic scaling behavior put forward here. The important point is that such interactions only induce finite-size correlations, whose length scale becomes negligible in the quantum critical limit, where critical quantum correlations develop a diverging length scale in the system.

\section{THE KITAEV QUANTUM WIRE SUBJECT TO DISSIPATION}

To provide evidence of the scaling laws put forward in Sec. III. we consider a Kitaev quantum wire defined by the Hamiltonian [10]

$$
\hat{H}_{\mathrm{K}}=-t \sum_{j=1}^{L}\left(\hat{c}_{j}^{\dagger} \hat{c}_{j+1}+\delta \hat{c}_{j}^{\dagger} \hat{c}_{j+1}^{\dagger}+\text { h.c. }\right)-\mu \sum_{j=1}^{L} \hat{n}_{j},
$$

where $\hat{c}_{j}$ is the fermionic annihilation operator on the $j$ th site of the chain, $\hat{n}_{j} \equiv \hat{c}_{j}^{\dagger} \hat{c}_{j}$ is the density operator, and $\delta>0$. We set $\hbar=1$, and $t=1$ as the energy scale.

The Hamiltonian (17) can be mapped into a spin- $1 / 2$ $\mathrm{XY}$ chain, by means of a Jordan-Wigner transformation. It undergoes a continuous quantum transition at $\mu=\mu_{c}=-2$, independently of $\delta$, between a disordered $\left(\mu<\mu_{c}\right)$ and an ordered quantum phase $\left(|\mu|<\left|\mu_{c}\right|\right)$. This transition belongs to the two-dimensional Ising universality class [5], characterized by the length-scale critical exponent $\nu=1$, related to the renormalizaton-group dimension $y_{\mu}=1 / \nu=1$ of the Hamiltonian parameter $\mu$ (more precisely of the difference $\bar{\mu} \equiv \mu-\mu_{c}$ ). The dynamic exponent associated with the unitary quantum dynamics is $z=1$. Moreover, the renormalization-group dimension of the fermionic operators $\hat{c}_{j}$ and $\hat{c}_{j}^{\dagger}$ is $y_{c}=1 / 2$, and that of the density operator $\hat{n}_{j}$ is $y_{n}=1$ [5].

In the following we fix $\delta=1$, such that the corresponding spin model is the quantum Ising chain

$$
\hat{H}_{\mathrm{Is}}=-\sum_{j} \hat{\sigma}_{j}^{(3)} \hat{\sigma}_{j+1}^{(3)}-g \sum_{j} \hat{\sigma}_{j}^{(1)},
$$

with $\hat{\sigma}_{j}^{(k)}$ being the Pauli matrices and $g=-\mu / 2$. In the following we prefer to stick with the Kitaev quantum wire, because the dissipation that we consider is more naturally defined for Fermi lattice gases.

We focus on the dynamic behavior of the Fermi lattice gas (17) close to its quantum transition, in the presence of homogeneous dissipation mechanisms following the Lindblad equation $(9)$. The dissipator $\mathbb{D}[\rho]$ is defined as a sum of local (single-site) terms of the form

$$
\mathbb{D}_{j}[\rho]=\hat{L}_{j} \rho \hat{L}_{j}^{\dagger}-\frac{1}{2}\left(\rho \hat{L}_{j}^{\dagger} \hat{L}_{j}+\hat{L}_{j}^{\dagger} \hat{L}_{j} \rho\right),
$$

where $\hat{L}_{j}$ denotes the Lindblad jump operator associated with the system-bath coupling scheme, and the index $j$ corresponds to a lattice site [thus replacing the index $O$ in Eqs. [10, (11)]. The onsite Lindblad operators $\hat{L}_{j}$ describe the coupling of each site with an independent bath $\mathcal{B}$, Fig. 1. We consider dissipation mechanisms associated with either particle losses (l) or pumping (p) [25, 26]:

$$
\hat{L}_{1, j}=\hat{c}_{j}, \quad \hat{L}_{\mathrm{p}, j}=\hat{c}_{j}^{\dagger} .
$$

The uniqueness of the eventual steady state has been proven for the above decay and pumping operators [2124. The choice of such dissipators turns out to be particularly convenient for the numerically analysis, allowing us to obtain results for quite large size of the Kitaev model, see below.

Our protocol starts from the ground state of $\hat{H}_{K}$ for a generic $\bar{\mu}_{i} \equiv \mu_{i}-\mu_{c}$, sufficiently small to stay within the critical regime. To address the competition between coherent and dissipative dynamics, we study the evolution after a quench of the Hamiltonian parameter to $\bar{\mu}$, and a simultaneous sudden turning on of the dissipation coupling $u$. To characterize te dynamic properties of the evo- 
lution described by the Lindblad equation, and in particular the corresponding asymptotic large-time behavior, we consider the fixed-time correlations

$$
\begin{aligned}
G_{p}(x, t) & =\operatorname{Tr}\left[\rho(t)\left(\hat{c}_{j}^{\dagger} \hat{c}_{j+x}^{\dagger}+\hat{c}_{j+x} \hat{c}_{j}\right)\right] \\
G_{c}(x, t) & =\operatorname{Tr}\left[\rho(t)\left(\hat{c}_{j}^{\dagger} \hat{c}_{j+x}+\hat{c}_{j+x}^{\dagger} \hat{c}_{j}\right)\right] \\
G_{n}(x, t) & =\operatorname{Tr}\left[\rho(t) \hat{n}_{j} \hat{n}_{j+x}\right]-\operatorname{Tr}\left[\rho(t) \hat{n}_{j}\right] \operatorname{Tr}\left[\rho(t) \hat{n}_{j+x}\right]
\end{aligned}
$$

where we used the space translation invariance of the system.

According to the scaling arguments reported in Sec. III for systems close to a continuous quantum transition, in particular for $\lambda \equiv|\bar{\mu}|^{-1} \rightarrow \infty$, these correlation functions are expected to develop the scaling laws given in Eqs. (12) and (13). The approach to the asymptotic behavior is foreseen to be controlled by power-law corrections. Relying on the analysis of closed systems undergoing quantum transition belonging to the two-dimensional Ising universality class, see Ref. [13], we generally expect $O\left(\lambda^{-1}\right)$ corrections, generally arising from analytic corrections to the nonlinear scaling fields [while scaling corrections arising from the leading irrelevant perturbation are more suppressed in the two-dimensional Ising universality class, as $O\left(\lambda^{-2}\right)$ ]. The numerical results reported in the next section nicely support these predictions.

\section{NUMERICAL RESULTS}

In this section we report numerical evidence of the scaling laws put forward in Sec. III for quantum wires in the presence of dissipation arising from incoherent particle losses and pumping. The choice of the dissipators 20 allow us to numerically solve the dynamic problem for systems with up to thousands of sites [27, 28. Indeed, the dynamics of the dissipative fermionic Kitaev chain (Fig. 1) can be written in terms of coupled linear differential equations, whose number scales linearly with the size $L$. We employ a fourth-order Runge-Kutta method in order to integrate them. Further details on the computation of the time trajectories from the Lindblad equation (9) are reported in Ref. [9].

In our analysis we always consider antiperiodic boundary conditions, i.e., such that $\hat{c}_{L+1}=-\hat{c}_{1}$, which turn out to be technically convenient for the calculations. However, since we always consider sufficiently large chains to be effectively in the thermodynamic limit (more precisely $L \gg \lambda$, where $\lambda=\bar{\mu}^{-1}$ ), the results that we present are not affected by this particular choice. The realization of the infinite-size limit is easily verified by comparing the numerics at fixed $\lambda$ and $u$, with increasing $L$. All the data presented here should be considered in the infinitesize limit with a great accuracy (finite-size effects are invisible on the scales of all the figures shown below).

Let us start by presenting some numerical outcomes for the correlation function $G_{p}(x, t)$, cf. Eq. 21a), in the presence of dissipation arising from incoherent particle losses. The curves shown in Fig. 2 have been obtained
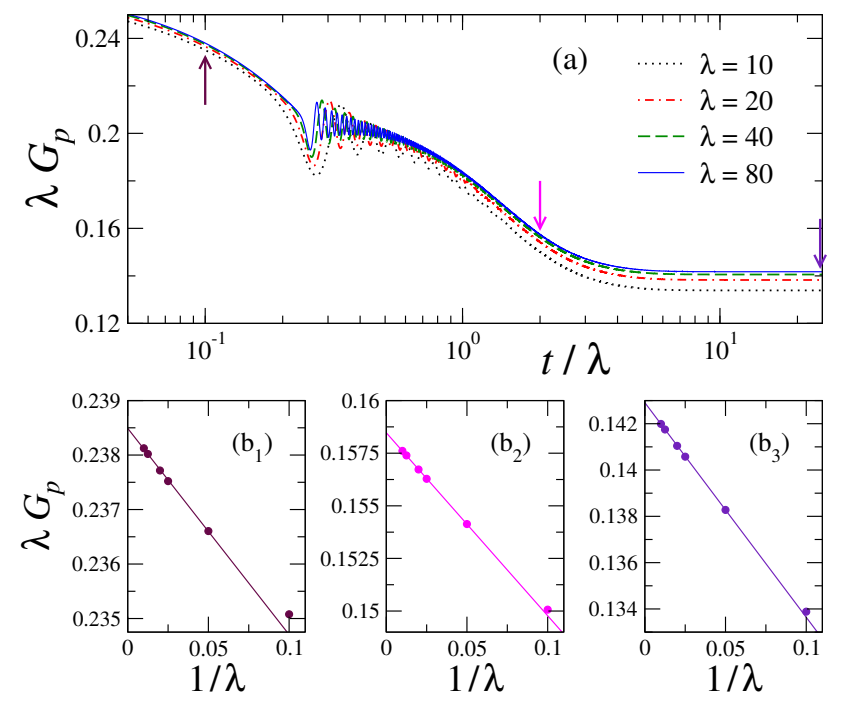

FIG. 2: (a) The correlation function $G_{p}(x, t)$ in Eq. 21a, multiplied by $\lambda$, versus the rescaled time $t / \lambda$. Different curves are for various values of $\lambda$, as indicated in the legend. Here we fix $x / \lambda=1$. The dissipation is induced by incoherent particle losses and its rescaled strength is kept constant, such that $u \lambda=1$. We also fix $\bar{\mu}_{i}=\bar{\mu}$ (no Hamiltonian quenches) and take $\bar{\mu}<0$ (i.e., approaching the critical point from the left side: $\mu<\mu_{c}$ ), which vary for each curve as $|\bar{\mu}|=\lambda^{-1}$, cf. Eq. (1). The curves approach a scaling function with increasing $\lambda$, thus supporting the scaling equation $12 .\left(b_{1}\right)$ $\left(b_{2}\right)-\left(b_{3}\right)$ The convergence with $\lambda$ of the various curves in panel (a), for fixed values of the rescaled time, $t / \lambda=0.1$, 2 , and 25, respectively [arrows in panel (a)]. Straight lines are fits $\propto \lambda^{-1}$ of numerical data (circles), whose extrapolation to $\lambda \rightarrow+\infty$ produces the values $0.2385,0.1585,0.1429$, respectively.

for the protocol with $\bar{\mu}_{i}=\bar{\mu}$ (no Hamiltonian quenches) and $\bar{\mu}<0$ (thus approaching the critical point from the left side: $\mu<\mu_{c}$ ), keeping the rescaled dissipation strength constant, such that $u \lambda^{z}=u \lambda=1$. The results in panel (a) clearly support the asymptotic dynamic scaling behavior put forward in Eq. 12 , i.e., for $\mu_{i}=\mu$,

$$
G_{p}(x, t ; \mu, u) \approx \lambda^{-1} \mathcal{G}_{p}(x / \lambda, t / \lambda, u \lambda) .
$$

Indeed the data for $\lambda G_{p}(x, t)$ versus $t / \lambda$ at fixed $x / \lambda=1$ appear to converge toward an asymptotic curve exhibiting a nontrivial behavior, when increasing $\lambda$. Notice also that the long-time limit of such curve for $G_{p}$ is different from zero, thus signaling the approach to a nontrivial stationary state. Moreover, as expected (see end of Sec. IV), such convergence is characterized by $O\left(\lambda^{-1}\right)$ corrections, as is visible from the bottom panels of Fig. 2, which focus on three different values of $t / \lambda: 0.1$ [panel $\left(b_{1}\right)$ ], 2 [panel $\left(\mathrm{b}_{2}\right)$ ], and 25 [panel $\left(\mathrm{b}_{3}\right)$ ].

We have extensively verified that the same scaling behavior of Eq. 22 can be observed for any value of the ratio $x / \lambda$, and of the rescaled dissipation strength $u \lambda$. Furthermore an analogous dynamic scaling is developed by the correlation function $G_{c}$ defined in Eq. 21b), as 

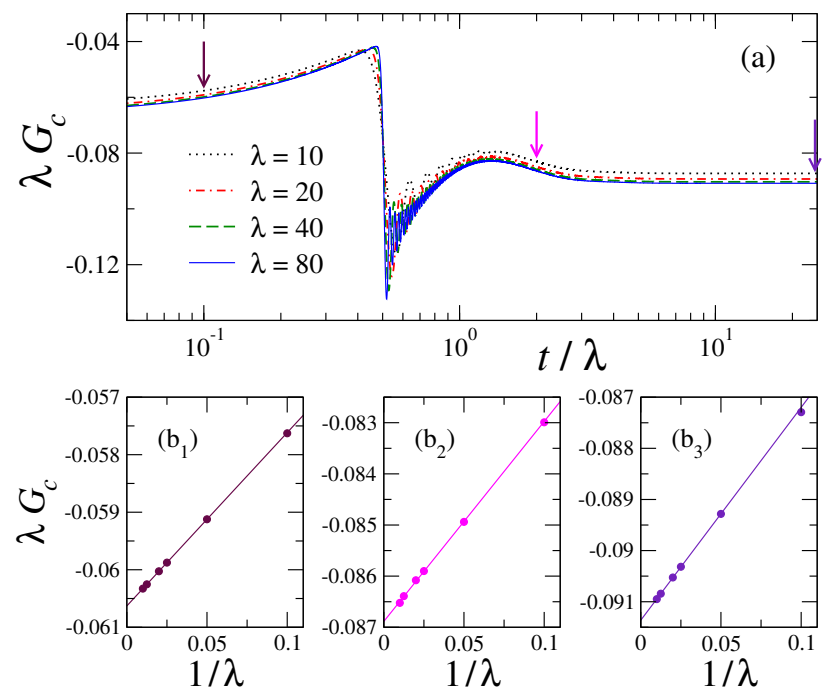

FIG. 3: Same as in Fig. 2, but for the correlation function $G_{c}(x, t)$ in Eq. 21b. All the various parameters are the same as in Fig. 2, except for the value of $x / \lambda$, which here is taken fixed and equal to 2 . The asymptotic values of the various curves in panel (a) for $\lambda \rightarrow+\infty$, extrapolated from a linear fit in $\lambda^{-1}$, are -0.0606 [panel $\left(b_{1}\right), t / \lambda=0.1$ ], -0.0869 [panel $\left.\left(\mathrm{b}_{2}\right), t / \lambda=2\right],-0.0914$ [panel $\left.\left(\mathrm{b}_{3}\right), t / \lambda=25\right]$.

explicitly shown in Fig. 3. For the sake of clarity in our presentation, in that figure we adopted the same framework and conventions of Fig. 2, and used the same set of parameters, with the exception of $x / \lambda$, which here has been set equal to 2 . Also in that situation we notice a remarkable agreement with the predicted $O\left(\lambda^{-1}\right)$ scaling for finite- $\lambda$ corrections (see bottom panels of Fig. 3).

As a further check of the dynamic scaling theory, Fig. 4 displays results for the density-density correlation function $G_{n}(x, t)$, cf. Eq. $\left.21 \mathrm{c}\right)$, for two different values of rescaled dissipation strength $u \lambda$. Again, they support the dynamic scaling behavior put forward in Sec. III. see in particular Eq. (12), keeping into account that the renormalization-group dimension of the density operator is now $y_{n}=1$. Therefore, for $\bar{\mu}_{i}=\bar{\mu}$,

$$
G_{n}(x, t ; \mu, u) \approx \lambda^{-2} \mathcal{G}_{n}(x / \lambda, t / \lambda, u \lambda),
$$

i.e., the product $\lambda^{2} G_{n}(x, t)$ is expected to approach a nontrivial asymptotic large- $\lambda$ scaling behavior in terms of the scaling variables $x / \lambda, t / \lambda$ and $u \lambda$, which eventually vanishes for $t / \lambda \rightarrow \infty$. The data in the two insets, which focus on a fixed rescaled time $t / \lambda=2$, reveal that the $1 / \lambda$ corrections to the scaling are, for this observable, superimposed to fluctuations which witness the complex oscillatory behavior of the scaling functions (compare the different scales in the $y$-axis of the main panels and of the insets).

Analogous scaling results can be obtained when approaching the critical point from the right side: $\mu>\mu_{c}$, thus $\bar{\mu}>0$, as shown by the curves reported in Fig. 5 for the three considered correlation functions. Comparing the results for the asymptotic stationary states with
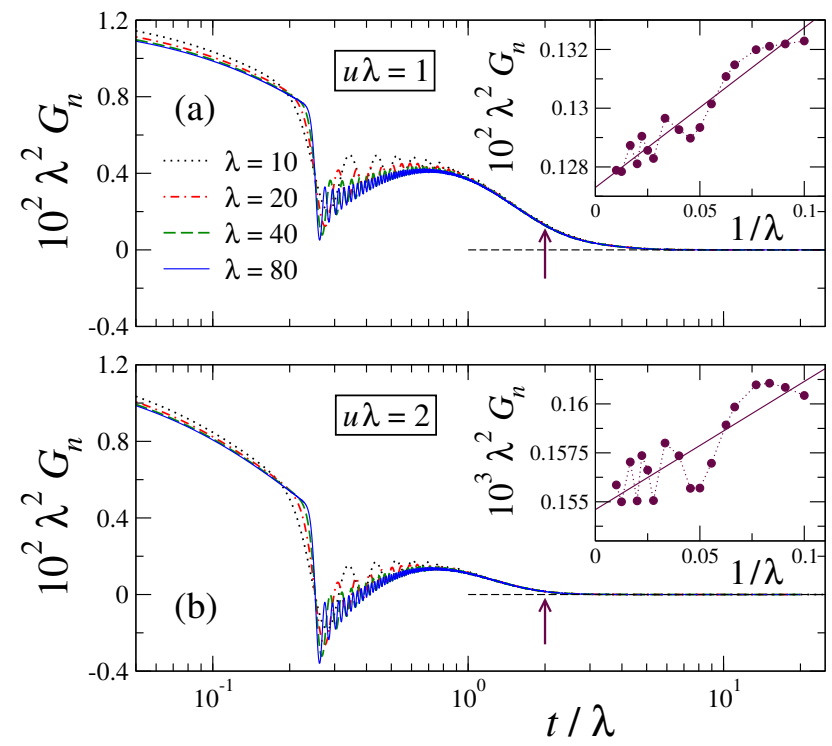

FIG. 4: The correlation function $G_{n}(x, t)$ in Eq. 21c), multiplied by $\lambda^{2}$, versus the rescaled time $t / \lambda$. Panel (a) is with a dissipation strength $u \lambda=1$, while panel (b) is with $u \lambda=2$. Here we fix $x / \lambda=1, \bar{\mu}_{i}=\bar{\mu}$, and take $\bar{\mu}=-1 / \lambda$. The horizontal dashed line indicates the zero value, which is reached asymptotically at long times, and is plotted as a guideline. The two insets show the convergence with $\lambda$ of the curves at $t / \lambda=2$ (arrow in the two main panels). The rather complex behavior of the large- $\lambda$ convergence reflects the oscillatory behavior of the curves as a function of $t / \lambda$, which becomes more evident on the scale of the main frames for $0.3 \lesssim t / \lambda \lesssim 1$. The dashed lines of the insets are drawn to guide the eyes (they suggest that the large- $\lambda$ limit approached by the data is $\approx 0.127$ for $u \lambda=1$ and $\approx 0.155$ for $u \lambda=2$ ).

those for $\mu<\mu_{c}$ at equal values of $|\bar{\mu}|$, see, in particular, Fig. 2(a), Fig. 3(a), and Fig. 4, we notice that while the absolute values of the rescaled correlation functions are the same when approaching the critical point from either side, the respective signs for the correlation $G_{c}$ are exchanged.

Let us now discuss more in detail the behavior of stationary states, which are approached in the large-time limit. Figure 6 shows that the quantities $\lambda G_{p, s}$ and $\lambda G_{c, s}$, obtained by taking the $t \rightarrow \infty$ limit of $G_{p}$ and $G_{c}$, approach an asymptotic large- $\lambda$ scaling form as a function of $x / \lambda$ and $u \lambda$ [cf. Eq. [13)]. Indeed, they show that

$$
G_{p / c, s}\left(x ; \mu_{i}, \mu, u\right) \approx \lambda^{-1} \Gamma_{p / c}(x / \lambda, u \lambda),
$$

as put forward in Eq. (13). Moreover, they appear to decay exponentially for sufficiently large distances, i.e.,

$$
\lambda G_{p / c, s} \sim \exp \left[-x / \xi_{s}\right]=\exp \left[-\frac{x / \lambda}{\xi_{s} / \lambda}\right],
$$

where $\xi_{s}$ defines a correlation length which depends on the dissipation strength, cf. Eq. (14). Therefore, Eq. 25) also implies the scaling behavior

$$
\xi_{s} \sim \lambda \equiv|\bar{\mu}|^{-\nu},
$$






FIG. 5: Results for $\lambda G_{p}(x, t)$ [panel (a)], $\lambda G_{c}(x, t)$ [panel (b)], and $\lambda^{2} G_{n}(x, t)$ [panel (c)], versus the rescaled time $t / \lambda$. The various system parameters are set as in Fig. 4(a), with the exception of the Hamiltonian control parameter $\bar{\mu}_{i}=\bar{\mu}$, with $|\bar{\mu}|=\lambda^{-1}$, which here has been chosen to be positive (i.e., approaching the critical point from the right side: $\mu>\mu_{c}$ ).

as predicted by the scaling equation 15 .

All the results presented so far have been obtained for $\mu_{i}=\mu$, thus in the absence of any Hamiltonian quench. Relaxing this assumption, we found that the stationary values approached by the various correlators in the longtime limit are independent of the initial condition of the protocol, in particular of the value of $\mu_{i} \neq \mu$, and thus obey the same asymptotic scaling behaviors discussed above. This is explicitly shown in Fig. 77, for the correlation function $G_{p}$. This fact is consistent with the observation that, for our choice of dissipators, the asymptotic stationary states are indeed unique.

We conclude our analysis by mentioning that completely analogous results can be obtained in the case the dissipative mechanism is related to a uniform local pumping, associated with the Lindblad operator $\hat{L}_{\mathrm{p}, j}$ in Eq. 20. (not shown).

\section{SUMMARY AND CONCLUSION}

We have investigated the effects of dissipation on the dynamics of many-body systems close to a continuous quantum phase transition, arising from the interaction with the environment, as for example sketched in Fig. 1. The latter is modeled through a class of dissipative mechanisms that can be effectively described by Lindblad equations for the density matrix of the system [6, 7, with
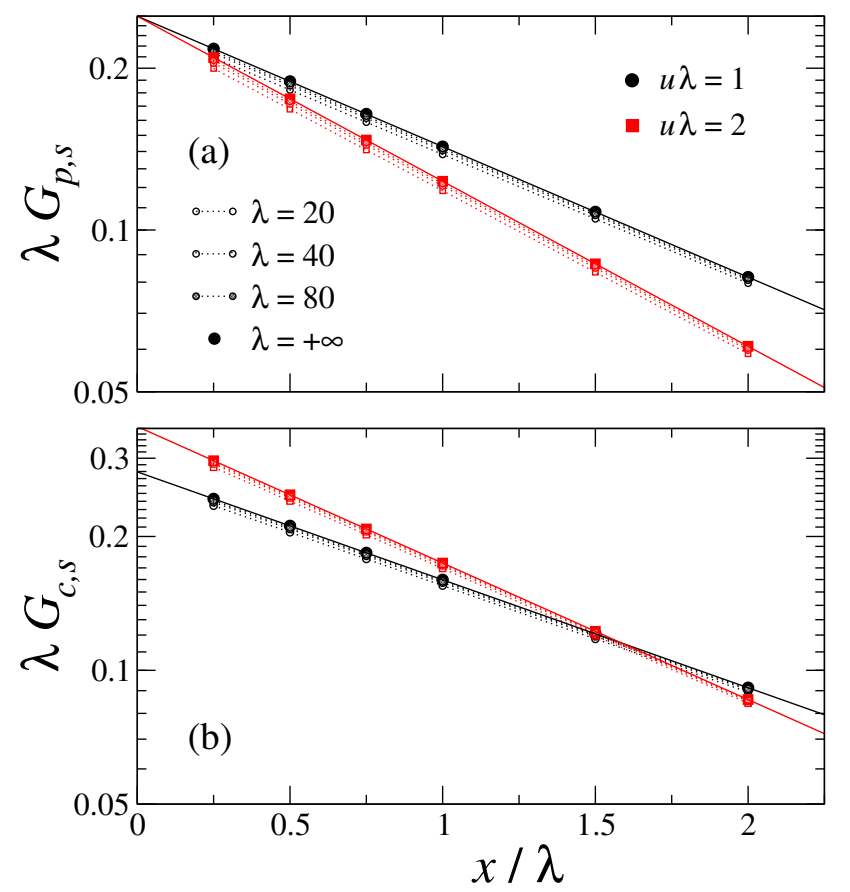

FIG. 6: Results for $\lambda G_{p, s}(x)$ [panel (a)] and $\lambda G_{c, s}(x)$ [panel (b)] in the large-time limit (checked numerically with great accuracy), as a function of the rescaled variable $x / \lambda$. Smaller and partially filled symbols stand for three specific values of $\lambda$, according to the legend, while fully filled symbols denote the values extrapolated for $\lambda \rightarrow+\infty$, that is, by extrapolating to zero the data at finite $\lambda^{-1}$ [see, e.g., type-(b) panels in Figs. 2 and 3. Black data sets and circles are for a dissipation strength $u \lambda=1$, while red data sets and squares are for $u \lambda=$ 2 . Here we always choose $\bar{\mu}<0$. Straight lines are exponential fits $G_{s} \sim e^{-(x / \lambda) /(\xi / \lambda)}$ of the extrapolated numerical data for $\lambda \rightarrow+\infty$. For both kinds of correlation functions, the obtained decay rates are $\xi / \lambda=1.789$ (for $u \lambda=1$ ) and $\xi / \lambda=$ 1.414 (for $u \lambda=2$ ), with a relative discrepancy smaller than $10^{-4}$, in support of the scaling law 15 .

local and homogenous Lindblad operators, such as those reported in Eqs. (9)-(11). This framework is of experimental interest, indeed the conditions for its validity are typically realized in quantum optical and c-QED implementations [1, 2, 8].

We have analyzed how homogenous dissipative mechanisms change the scaling laws of closed systems at quantum transitions. For this purpose, we have considered a relatively simple dynamic protocol: the quantum manybody system is initialized, at $t=0$, into the ground state of the Hamiltonian $\hat{H}\left(\mu_{i}\right)$ for a given parameter $\mu_{i}$; then for $t>0$ the system evolves according to the Lindblad equation (9), where the coherent driving is provided by the Hamiltonian $\hat{H}(\mu)$, and the dissipation arising from the system-bath interaction is effectively described by the dissipator $\mathbb{D}[\rho]$ with a fixed coupling strength $u$.

The large-time stationary state is usually noncritical, even when the Hamiltonian parameters are critical. However, we identified a low-dissipation regime where the dis- 


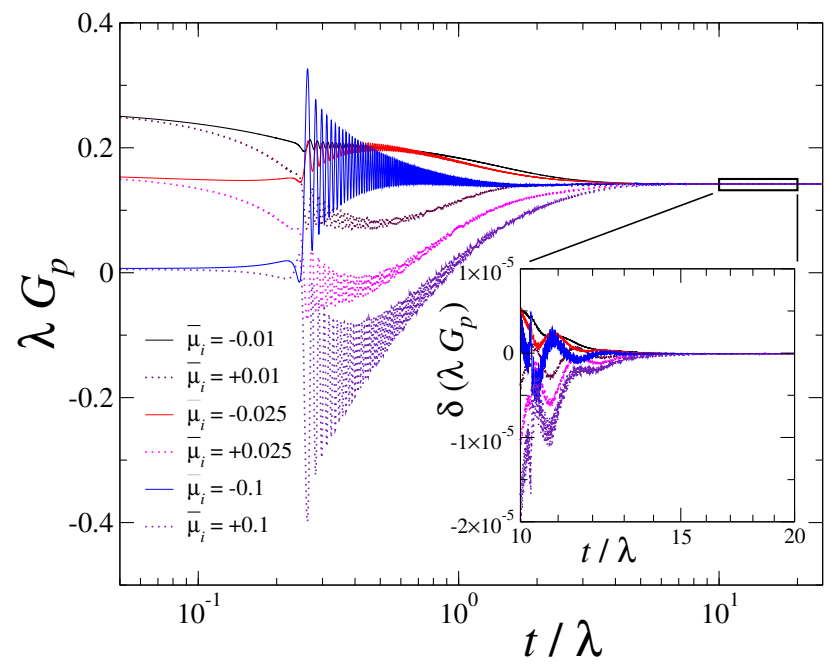

FIG. 7: The correlation function $G_{p}(x, t)$ in Eq. 21a), multiplied by $\lambda$, versus the rescaled time $t / \lambda$. Here we fix $x / \lambda=1$, $u \lambda=1$, and $\bar{\mu}=-0.01$ (corresponding to $\lambda=100$ ). The various curves correspond to different initial conditions, determined by the ground states of the Kitaev Hamiltonian with changing $\bar{\mu}_{i}$, being either negative (i.e., $\mu_{i}<\mu_{c}$ ) or positive $\left(\mu_{i}>\mu_{c}\right)$ (see the legend). The inset provides a magnification of the numerical data for $10 \leq t / \lambda \leq 20$ (box in the main frame), after subtracting the asymptotic value for $t / \lambda \rightarrow \infty$.

sipation is sufficiently small to compete with the coherent evolution driven by the critical Hamiltonian, leading to stationary states which present critical behaviors depending also on the strength of the dissipation coupling. The above mentioned regime is generally realized when the dissipation parameter $u$ scales as the gap $\Delta$ of the Hamiltonian of the many-body system, i.e., $u \sim \Delta$. Therefore it is a low-dissipation regime, in that the gap gets suppressed at the quantum transition, as $\Delta \sim \xi^{-z}$, where $\xi$ is the large length scale developed by the critical correlation functions. This reflects the fact that at a quantum transition the perturbation arising from dissipation is always relevant, such as the temperature at equilibrium [5, 12, 13]. This also means that, when $u \gg \Delta$, critical fluctuations do not survive to the dissipation.

We argue that, under such low-dissipation conditions, open many-body systems develop dynamic scaling laws, which apply to the time evolution described by the Lindblad equation (9), and in particular to the stationary states arising in the large-time limit (see Sec. III). Analogous to the scaling laws of closed systems at quantum transitions, the dynamic scaling behavior in the presence of dissipation is expected to be largely independent of the microscopic details of both coherent and dissipative drivings, as for the critical behavior of closed systems, which only depends on the universality class of the quantum transition. Further investigation is called for, to assess the actual extension of the universality of the dynamic scaling functions with respect to the properties of the dissipative mechanisms.

The dynamic scaling laws obtained in this paper apply to complementary regimes with respect to those recently reported in Ref. [9], where finite systems of linear size $L$ and dynamic behavior for relatively small times $t \sim L^{z}$ were considered, thus not including the large-time stationary regime. On the other hand, here we focused on infinite-size systems, for which we managed to derive scaling laws extending to the large-time limit of the evolution described by the Lindblad equation, thus valid for the corresponding stationary states.

The dynamic scaling scenario has been checked within fermion wires, cf. Eq. (17), in the presence of dissipation due to local incoherent pumping and decay, which are described by the Lindblad operators reported in Eq. 200. Our numerical analysis supports our general, phenomenological, dynamic scaling theory addressing the competition between coherent dynamics and dissipation at a continuous quantum transition. Further checks of the dynamic scaling behaviors may turn out to be interesting for other many-body systems at quantum transitions, possibly belonging to different universality classes, and/or dissipation mechanisms, including nonlocal ones 29. 31 .

The arguments leading to this scenario are quite general. We believe that analogous phenomena should develop in any homogeneous $d$-dimensional many-body system at a continuous quantum transition, whose Markovian interaction with the environment can be described by local or extended dissipators within a Lindblad equation (9). These arguments should also apply to nonMarkovian system-bath couplings 32] (not described by Lindblad equations), replacing $u$ with the parameter controlling the decay rate.

We finally mention that some experimental breakthroughs were recently achieved in the control of dissipative quantum many-body dynamics, through different platforms, such as Rydberg atoms or c-QED technology. Quantum critical behaviors in such out-of-equilibrium context were reported in Refs. [19, 33, 34].
[1] A. A. Houck, H. E. Türeci, and J. Koch, On-chip quantum simulation with superconducting circuits, Nat. Phys. 8, 292 (2012).

[2] M. Müller, S. Diehl, G. Pupillo, and P. Zoller, Engineered open systems and quantum simulations with atoms and ions, Adv. At. Mol. Opt. Phys. 61, 1 (2012).

[3] I. Carusotto and C. Ciuti, Quantum fluids of light, Rev.
Mod. Phys. 85, 299 (2013).

[4] M. Aspelmeyer, T. J. Kippenberg, and F. Marquardt, Cavity optomechanics, Rev. Mod. Phys. 86, 1391 (2014).

[5] S. Sachdev, Quantum Phase Transitions, (Cambridge University, Cambridge, England, 1999).

[6] H.-P. Breuer and F. Petruccione, The Theory of Open Quantum Systems (Oxford University Press, New York, 
2002).

[7] A. Rivas and S. F. Huelga, Open Quantum System: An Introduction (SpringerBriefs in Physics, Springer, 2012).

[8] L. M. Sieberer, M. Buchhold, and S. Diehl, Keldysh field theory for driven open quantum systems, Rep. Prog. Phys. 79, 096001 (2016).

[9] D. Nigro, D. Rossini, and E. Vicari, Competing coherent and dissipative dynamics close to quantum criticality, Phys. Rev. A 100, 052108 (2019).

[10] A. Yu. Kitaev, Unpaired Majorana fermions in quantum wires, Phys. Usp. 44, 131 (2001).

[11] D. Rossini and E. Vicari, Scaling of decoherence and energy flow in interacting quantum spin systems, Phys. Rev. A 99, 052113 (2019); E. Vicari, Decoherence dynamics of qubits coupled to systems at quantum transitions, Phys. Rev. A 98, 052127 (2018).

[12] S. L. Sondhi, S. M. Girvin, J. P. Carini, and D. Shahar, Continuous quantum phase transitions, Rev. Mod. Phys. 69, 315 (1997).

[13] M. Campostrini, A. Pelissetto, and E. Vicari, Finite-size scaling at quantum transitions, Phys. Rev. B 89, 094516 (2014).

[14] A. Pelissetto, D. Rossini, and E. Vicari, Dynamic finitesize scaling after a quench at quantum transitions, Phys. Rev. E 97, 052148 (2018).

[15] D. Nigro, D. Rossini, and E. Vicari, Scaling properties of work fluctuations after quenches near quantum transitions, J. Stat. Mech. (2019) 023104.

[16] G. Lindblad, On the generators of quantum dynamical semigroups, Commun. Math. Phys. 48, 119 (1976).

[17] V. Gorini, A. Kossakowski, and E. C. G. Sudarshan, Completely positive dynamical semigroups of N-level systems, J. Math. Phys. 17, 821 (1976).

[18] J. Jin, A. Biella, O. Viyuela, L. Mazza, J. Keeling, R. Fazio, and D. Rossini, Cluster mean-field approach to the steady-state phase diagram of dissipative spin systems, Phys. Rev. X 6, 031011 (2016).

[19] M. Fitzpatrick, N. M. Sundaresan, A. C. Y. Li, J. Koch, and A. A. Houck, Observation of a Dissipative Phase Transition in a One-Dimensional Circuit QED Lattice, Phys. Rev. X 7, 011016 (2017).

[20] S. Yin, P. Mai, and F. Zhong, Nonequilibrium quantum criticality in open systems: The dissipation rate as an additional indispensable scaling variable, Phys. Rev. B 89, 094108 (2014); S. Yin, C.-Y. Lo, and P. Chen, Scaling behavior of quantum critical relaxation dynamics of a system in a heat bath, Phys. Rev. B 93, 184301 (2016).

[21] E. B. Davies, Quantum stochastic processes II, Commun. Math. Phys. 19, 83 (1970); Quantum stochastic processes, Commun. Math. Phys. 15, 277 (1969).

[22] D. E. Evans, Irreducible Quantum Dynamical Semigroups, Commun. math. Phys. 54, 293 (1977).

[23] S. G. Schirmer and X. Wang, Stabilizing open quantum systems by Markovian reservoir engineering, Phys. Rev. A 81, 062306 (2010).

[24] D. Nigro, On the uniqueness of the steady-state solution of the Lindblad-Gorini-Kossakowski-Sudarshan equation, J. Stat. Mech. (2019) 043202.

[25] B. Horstmann, J. I. Cirac, and G. Giedke, Noise-driven dynamics and phase transitions in fermionic systems, Phys.Rev. A 87, 012108 (2013).

[26] M. Keck, S. Montangero, G. E. Santoro, R. Fazio, and D. Rossini, Dissipation in adiabatic quantum computers: lessons from an exactly solvable model, New. J. Phys. 19,
113029 (2017)

[27] T. Prosen, Third quantization: a general method to solve master equations for quadratic open Fermi systems, New J. Phys. 10, 043026 (2008).

[28] V. Eisler, Crossover between ballistic and diffusive transport: the quantum exclusion process, J. Stat. Mech. (2011) P06007.

[29] F. Verstraete, M. Wolf, and J. I. Cirac, Quantum computation and quantum-state engineering driven by dissipation, Nat. Phys. 5, 633 (2009).

[30] S. Diehl, E. Rico, M. A. Baranov, and P. Zoller, Topology by dissipation in atomic quantum wires, Nat. Phys. 7, 971 (2011).

[31] A. C. Y. Li, F. Petruccione, and J. Koch, Resummation for nonequilibrium perturbation theory and application to open quantum lattices, Phys. Rev. X 6, 021037 (2016).

[32] I. de Vega and D. Alonso, Dynamics of non-Markovian open quantum systems, Rev. Mod. Phys. 89, 015001 (2017).

[33] C. Carr, R. Ritter, C. G. Wade, C. S. Adams, and K. J. Weatherill, Nonequilibrium phase transition in a dilute Rydberg ensemble, Phys. Rev. Lett. 111, 113901 (2013).

[34] T. Tomita, S. Nakajima, I. Danshita, Y. Takasu, and Y. Takahashi, Observation of the Mott insulator to superfluid crossover of a driven-dissipative Bose-Hubbard system, Sci. Adv. 3, e1701513 (2017). 